\title{
QCM-D study of time-resolved cell adhesion and detachment: Effect of surface free energy on eukaryotes and prokaryotes
}

\author{
Derick Yongabi ${ }^{\text {a* }}$, Mehran Khorshid a , Alessia Gennaro a, Stijn Jooken a , Sam Duwé b, \\ Olivier Deschaume ${ }^{a}$, Patricia Losada-Pérez ${ }^{c}$, Peter Dedecker ${ }^{b}$, Carmen Bartic ${ }^{a}$, \\ Michael Wübbenhorst ${ }^{a}$, Patrick Wagner ${ }^{\text {a }}$
}

a KU Leuven, Department of Physics and Astronomy, Laboratory for Soft Matter and Biophysics, Celestijnenlaan 200 D, B-3001 Leuven, Belgium

${ }^{\mathrm{b}}$ KU Leuven, Department of Chemistry, Laboratory for Nanobiology, Celestinenlaan $200 \mathrm{G}$, B-3001, Leuven, Belgium

${ }^{c}$ Université Libre de Bruxelles (ULB), Experimental Soft Matter and Thermal Physics Group, Campus La Plaine, CP223, Boulevard du Triomphe, 1050 Brussels, Belgium.

\section{* Corresponding author}

Drs. Derick Yongabi

KU Leuven

Laboratory for Soft-Matter and Biophysics

Celestijnenlaan 200 D

B-3001 Leuven, Belgium

Tel : +3216376874

E-mail: derick.yongabi@,kuleuven.be 
Section I: $\quad$ Surface free energy determination by the Owens, Wendt, Rabel and Kaelble (OWRK) method.

The contact angle is related to interfacial energies through Young's equation(Eq. 1).

$$
\gamma l v \cos \theta=\gamma s v-\gamma s l \quad \text { Eq. } 1
$$

Here, $\theta$ is the contact angle and $\gamma_{\mathrm{lv}}, \gamma_{\mathrm{sv}}, \gamma_{\mathrm{sl}}$, are the interfacial energies of liquid/vapor, solid/vapor, and solid/liquid phases, respectively. A representation of vapor-liquid-solid interfaces is shown in Figure S1. The liquid surface tension, $\gamma_{1 v}$, is known, but $\gamma_{\mathrm{sl}}$ is an unknown, which results from the solid surface tension $\left(\gamma_{\mathrm{sv}}\right)$, liquid surface tension $\left(\gamma_{\mathrm{lv}}\right)$ and the interactions between the two phases. The OWRK model assumes that these interactions are the geometric mean of all polar and dispersive parts (Eq. 2), resulting in the modified Young's equation of Eq. 3.

$$
\begin{array}{cc}
\gamma_{s l}=\gamma l v+\gamma_{\gamma v}-2(\sqrt{\gamma l v \gamma s v}+\sqrt{\gamma l v \gamma s v}) & \text { Eq. } 2 \\
\frac{\gamma l v(\cos \theta+1)}{2 \sqrt{\gamma l v}}=\sqrt{\gamma s v} \cdot \sqrt{\gamma l v / \frac{n}{\gamma l v}}+\sqrt{\gamma s v} & \text { Eq. } 3
\end{array}
$$

The OWRK method employs this relationship (Eq. 3) to determine the surface free energy as the sum of the polar and dispersive components using contact angles measured with at least two different liquids with known polar and dispersive surface tensions (Owens and Wendt, 1969, Żenkiewicz, 2007). 


\section{Section II: $\quad$ Supporting figures}

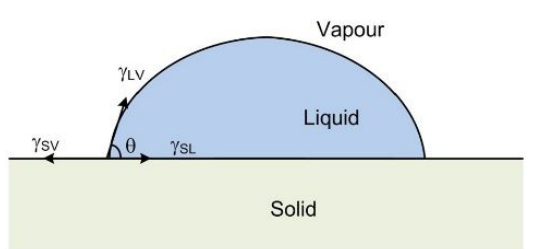

Figure S1: Schematic representation of the solid/liquid/vapour interphase.
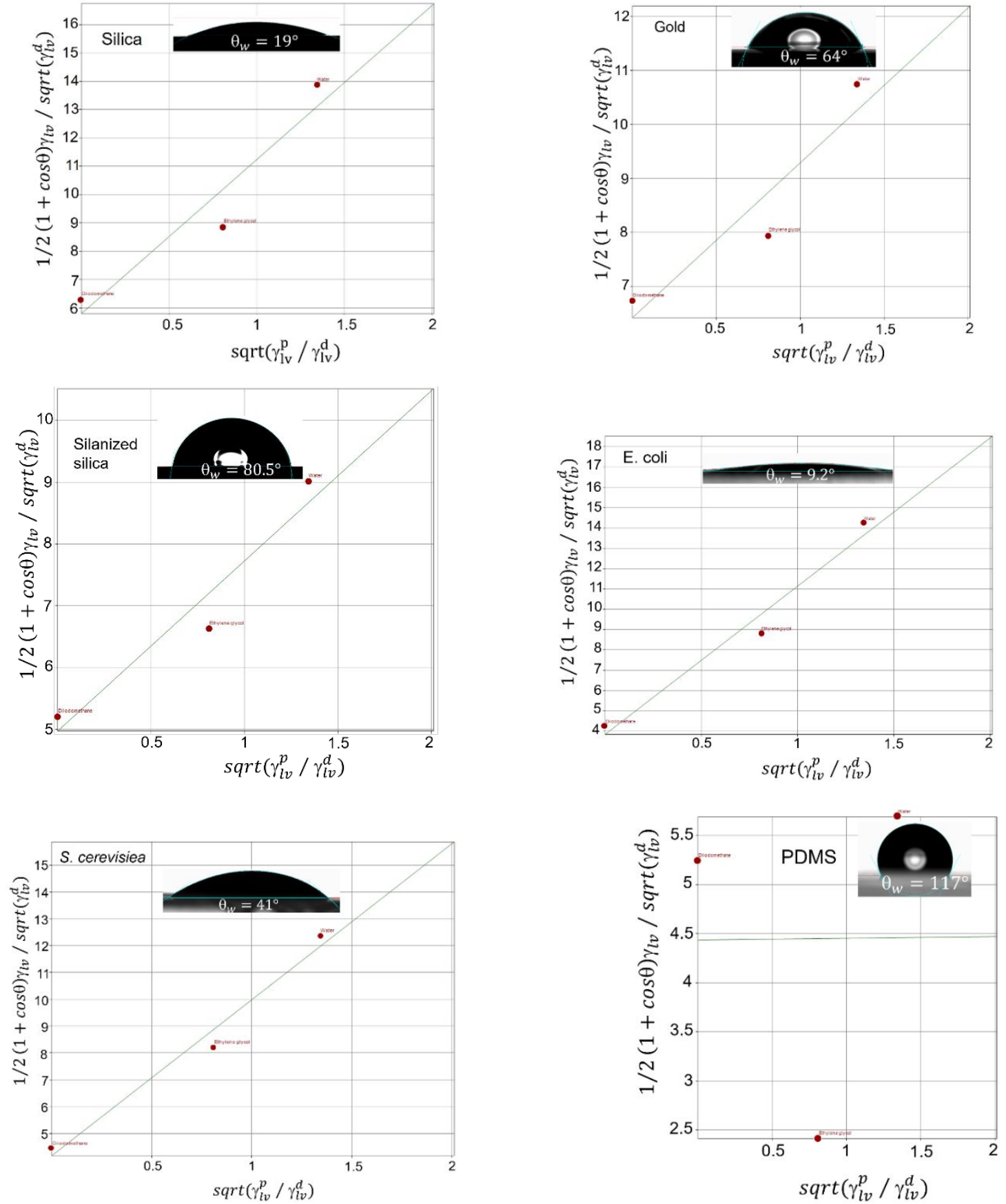

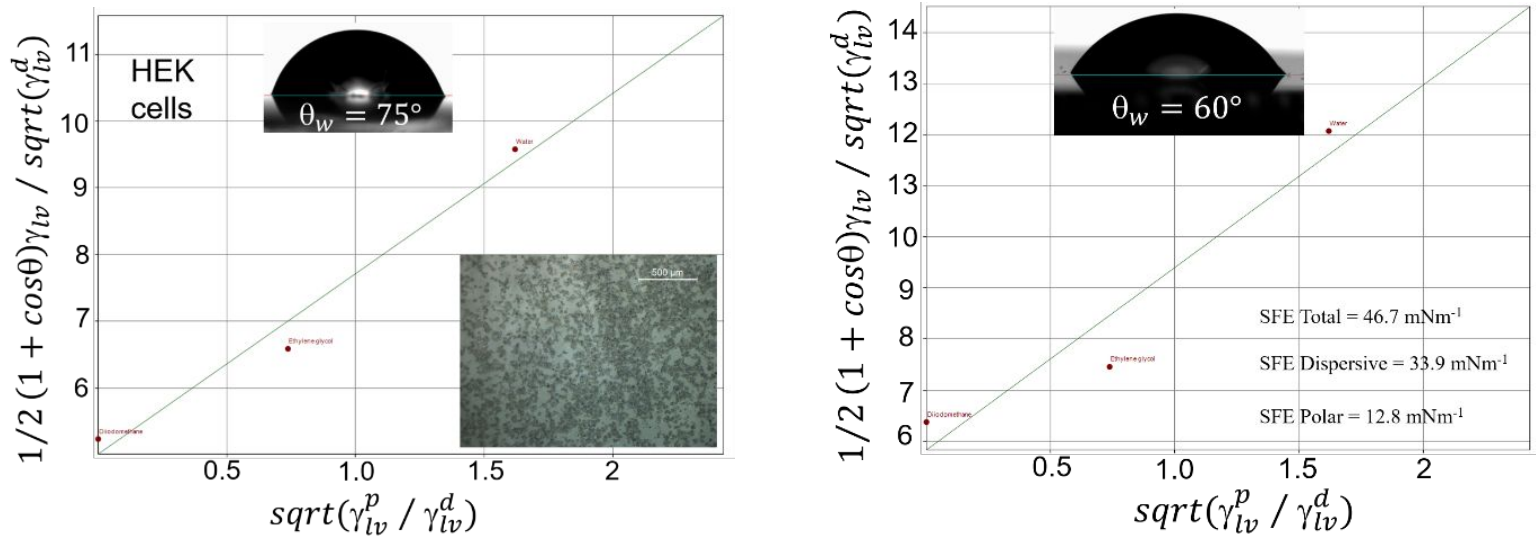

Figure S2: Surface free energy plots for silica, gold, silanized silica, E. coli, S. cerevisiae, HEK cells, cell culture flask and PDMS with inserts showing representative water contact angle measurements.
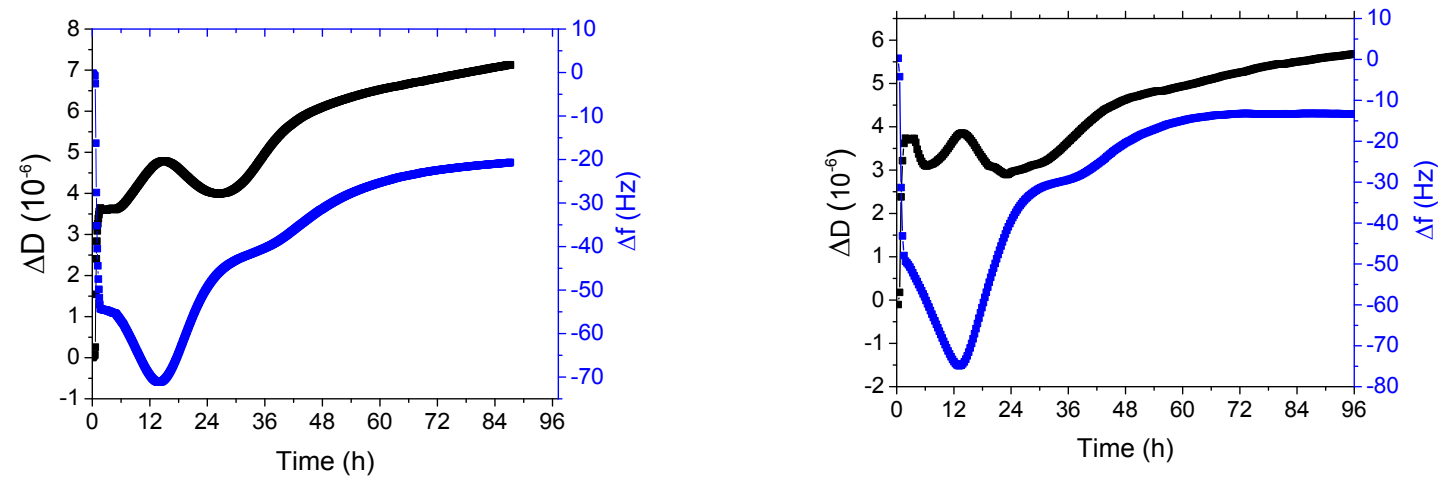

Figure S3: Time-dependent $\Delta D$ and $\Delta$ f plots for repeated yeast cell measurement on silica for a cell concentration of $1.6 \times 10^{6}$ cells $/ \mathrm{ml}$. All plots are based on the $7^{\text {th }}$ overtone.
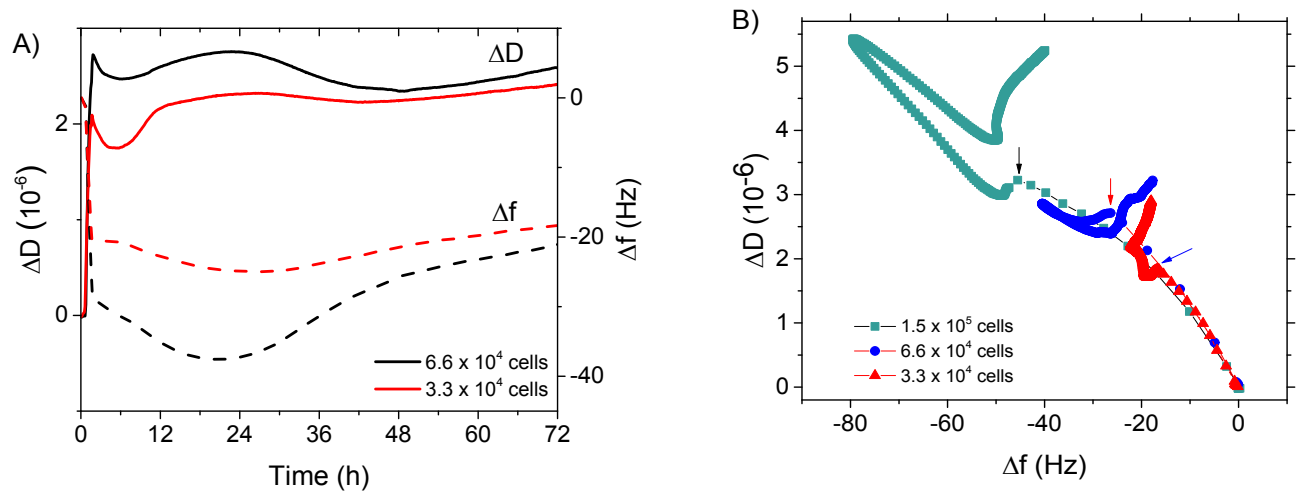

Figure S4: Time-dependent $\Delta D$ and $\Delta$ f plots from yeast cell measurement on silica for cell concentrations of 6.6 $\times 10^{4}$ and $3.3 \times 10^{5}$ cells $/ \mathrm{ml}$. All plots are based on the $7^{\text {th }}$ overtone $(A)$. Adhesion fingerprints of $S$. cerevisiae adhesion on silica for three cell concentrations $1.6 \times 10^{5}, 6.6 \times 10^{4}$ and $3.3 \times 10^{4} \mathrm{cell} / \mathrm{s} / \mathrm{m}$. 

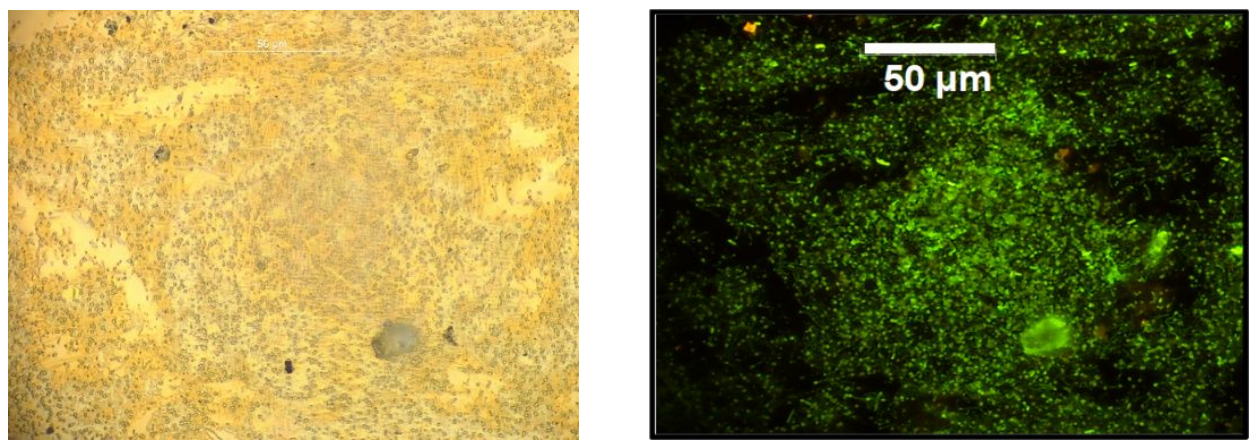

Figure S5: Left: Brightfield image of the rinsed gold surface after more than 24 hours adhesion monitoring, displaying structures clearly in the size range of E. coli. This indicates that the structural integrity of the cells is preserved and confirms that the fluorescence displayed on the right panel (Figure $\mathbf{B}$ in the main text) is from $E$. coli. 